\title{
A system of complementary genes in hybrids between Drosophila koepferae and D. buzzatii: A Markov chain model allows to make inferences about their number and relationships
}

\author{
Antonio R. Carvajal* \\ Departamento de Biología Celular y Molecular, Facultad de Ciencias, \\ Universidad de A Coruña, Campus da Zapateira \\ S/N, 15071 A Coruña, Spain.
}

(Received 26 February 2001, accepted 15 September 2001)

\begin{abstract}
In backcrosses between $D$. koepferae and $D$. buzzatii, the disruption of a system of species-specific complementary factors brings about hybrid male inviability. This system consists of a lethal factor, $h m i$-1, linked to the $\mathrm{X}$ chromosome of $\mathrm{D}$. koepferae, and several conspecific autosomal suppressors. However, hmi-1 hybrid males can also be rescued by factors present in some strains of $D$. buzzatii. The present work aims to estimate the number of $h m i-1$ suppressors in one of these strains by means of Markov chains. The obtained results allow discarding models with one or more chromosomes having independent suppressor effect. On the other hand, models having $n$ chromosomes that interact in groups of $r$, being $1<r \leq n$, to produce rescue effect, provide good approximations to the observed results. The best fit to the data is obtained with four or five chromosomes with suppressor effect, interacting epistatically in groups of three to rescue the viability of $h m i-1$ males.
\end{abstract}

\section{INTRODUCTION}

The genetic study of postzygotic reproductive isolation has frequently revealed an underlying complex genetic basis. Hence, the information accumulated in the past few years suggests a more complex genetics basis for postzygotic reproductive isolation than had been initially supposed (Wu \& Palopoli, 1994; Nei \& Zhang, 1998).

In hybrids between the sibling species $D$. buzzatii and $D$. koepferae, both sexes are more or less equally viable in the $\mathrm{F}_{1}$. However, in backcrosses to D. buzzatii, hybrid males are frequently inviable, apparently because of interespecific genetic incompatibilities that are cryptic in the $F_{1}$. This is the classical pattern of $F_{2}$ breakdown associated with coadapted gene complexes (Carson \& Templeton, 1984). In a previous work (Carvajal et al., 1996) a cytological region called $h m i-1$ was localised in the $\mathrm{X}$ chromosome of $D$. koepferae that, when introgressed in heterozygous condition in $D$. buzzatii, produces male inviability. Autosomal regions from $D$. koepferae were also localised that suppressed the inviability effect associated with $h m i-1$ when cointrogressed. These results apparently unveil a system of species-specific complemen-

Edited by Masa-Toshi Yamamoto

* Corresponding author. E-mail: acraaj@mail2.udc.es tary factors involved in a X-autosome interaction in $D$. koepferae that, when disrupted in backcross hybrids by recombination with the genome of its sibling $D$. buzzatii, brings about hybrid male inviability. However, there is still the possibility that some populations of $D$. buzzatii may harbour hybrid male rescue factors, similarly to what has been found in the melanogaster complex (Hutter et al., 1990). The present work shows the results of the introgression of $h m i-1$ into several $D$. buzzatii strains, and demonstrates that there is indeed a polymorphism for hybrid male rescue factors (suppressors of $h m i-1$ ). The number of suppressors and their mode of action in one of these strains are assessed by means of Markov chain models. As we will see, this kind of models allows making inferences about the expected proportion of backcross lines with viability rescue at any generation, and hence the proportion of rescued males. In order to do such predictions it is necessary to know both, the initial distribution of suppressors and the transition probability matrix i.e. the matrix of probabilities for shift from one state to another. In our case the unknown factors will be just these, the different types of initial distributions and their transition probability matrix corresponding to different models of suppressors which could explain the observed data. 


\section{MATERIAL AND METHODS}

Drosophila species $D$. buzzatii and D. koepferae are two sibling species of the repleta group that coexist in many of the arid and semiarid zones of Andean Bolivia and Northwest Argentina (Ruiz, Fontdevila \& Wasserman, 1982; Fontdevila et al., 1988). Both species have the standard $D$. repleta group polytene karyotype consisting in five rod-like chromosomes and a tiny dot-like chromosome. Number 1 corresponds to the X, numbers $2-5$ to the long acrocentric autosomes, and number 6 to the dot.

Stocks Three different kinds of stocks were used in the experiment described in this paper. (1) Five strains of $D$. buzzatii San Luis (buzSL): buzSL-5 and buzSL-6 (Naveira \& Fontdevila, 1991), founded by single wild-inseminated females collected at Serra San Luis (Argentina) and maintained by mass culturing; buzSL-101, with a white $(w)$ mutant fixed in homozygous condition in a genetic background of buzzatii San Luis (Naveira \& Fontdevila, 1991); buzSL-In(4)s, fixed for $4 s$, a natural population inversion of chromosome 4 (Ruiz \& Fontdevila, 1981); and buzSLIn(5)1, fixed for an inversion of chromosome 5 produced in the laboratory (Naveira \& Fontdevila, 1985). (2) A hybrid strain, buzSL-Xd[hmi-1, $\left.w^{+}\right]$(Carvajal et al., 1996), with $h m i-1$ introgressed in heterozygous condition together with the wild-type allele of white $\left(w^{+}\right)$from $D$. koepferae, on the genetic background of buzSL-101. (3) A set of $D$. buzzatii strains coming from the collection of Universidad Autónoma de Barcelona: buzDF, Dean Funes (Argentina); buzC, Carboneras (Spain); buzP, Plasencia (Spain); buzPR, Puebla del Río (Spain); and buzM, Mazarrón (Spain). All of them founded by single wildinseminated females collected at the respective localities and maintained by mass culturing.

Strain buzSL-Xd $\left[h m i-1, w^{+}\right]$is maintained by individual crosses of hybrid females (wild-type, red eyes) with buzSL101 males (white eyes), and selection of the adult offspring from those females that, according to the analysis of polytene chromosomes in third instar larvae (Naveira et al., 1986), still contained the $h m i-1-w^{+}$gametic association. Recombination distance between $h m i-1$ and white is $4 \mathrm{cM}$ (Carvajal et al., 1996). Therefore, 96\% of $w^{+}$male descendants from these crosses are expected to be carriers of $h m i-1$. This factor behaves as a fully penetrant lethal for males during pupal stages, so that the expected percentage of adult $w^{+}$males with respect to all $w^{+}$individuals is approximately $4 \%(0.04 / 1.04)$ in these maintenance crosses (Carvajal et al., 1996).

Crossing protocol Different $\mathrm{F}_{1}$ offsprings were obtained by crossing individual $w^{+}$buzSL-Xd[hmi-1, $\left.w^{+}\right]$ females with males from different buzzatii strains (table 1). Some of these crosses produced unusually high fre-
Table 1. Values of $\bar{m}$ (mean of the frequency of $w^{+}$hybrid males relative to $w^{+}$hybrid males plus females) from individual crosses between $w^{+}$buzSL-Xd $\left[h m i-1, w^{+}\right]$females and males from different buzzatii strains. Each kind of cross is designated with the name of the buzzatii male strain. Homogeneity $\left(\lambda_{\mathrm{H}}\right)$ and goodness of fit chi square with respect to the null hypothesis $\mu=0.04$ $\left(\lambda_{0.04}\right)$ are also given. Sample size is given in parenthesis in the column of.

\begin{tabular}{lllrc}
\hline \hline Cross & $\bar{m}$ (sample size) & $\lambda_{\mathrm{H}}$ & $\mathrm{df}$ & $\lambda_{0.04}$ \\
\hline buzSL-101 & $0.05(168)$ & $18.95 \mathrm{~ns}$ & 21 & $0.49 \mathrm{~ns}$ \\
buzSL-5 (1) & $0.41(787)$ & $30 \mathrm{~ns}$ & 20 & $4096^{* * *}$ \\
buzSL-5 (2) & $0.38(177)$ & $30.6^{* * *}$ & 8 & $274.6^{* * * *}$ \\
buzSL-6 & $0.05(99)$ & $3.58 \mathrm{~ns}$ & 6 & $0.27 \mathrm{~ns}$ \\
buzSL-In(4)s $(1)$ & $0.14(80)$ & $19.5^{* *}$ & 6 & $17.15^{* * *}$ \\
buzSL-In(4)s $(2)$ & $0.05(231)$ & $11.7 \mathrm{~ns}$ & 16 & $0.84 \mathrm{~ns}$ \\
buzSL-In(5)1 & $0.03(262)$ & $25.6 \mathrm{~ns}$ & 22 & $0.61 \mathrm{~ns}$ \\
buzDF & $0.12(150)$ & $15.39 \mathrm{~ns}$ & 10 & $24.17^{* *}$ \\
buzC & $0.08(72)$ & $13.27 \mathrm{~ns}$ & 8 & $3.52 \mathrm{~ns}$ \\
buzP & $0.07(71)$ & $7.99 \mathrm{~ns}$ & 6 & $1.71 \mathrm{~ns}$ \\
buzPR & $0.065(46)$ & $8.05 \mathrm{~ns}$ & 6 & $0.76 \mathrm{~ns}$ \\
buzM & $0.1(104)$ & $12.6 \mathrm{~ns}$ & 10 & $8.54^{* *}$ \\
\hline
\end{tabular}

ns: $P>0.05 ; *: 0.05>P>0.01 ; * *: 0.01>P>0.001 ; * * *: P<0.001$.

quencies of $w^{+}$males (see Results and table 1), therefore evidencing a rescue activity of $h m i-1$ hybrids by factors from respective strains. As will be shown in Results, crosses with strain buzSL-5 consistently produced high rescue frequencies, and were then chosen to carry out backcrosses in order to assess the maximum number of chromosomes with $h m i$ - 1 suppressors effect present in this strain (Fig. 1).

In our model, the genotype of buzSL-Xd[hmi-1, $\left.w^{+}\right]$ females will be represented as $L / L^{-} ; n\left(S u^{-}\right) / n\left(S u^{-}\right)$, and that of buzSL-5 males as $\mathrm{L}^{-} / \mathrm{Y} ; n(S u) / n(S u)$. In this notation, $L$ represents the lethal $h m i-1, S u$ is a suppressor allele of the $h m i-1$ effect, $n$ represents the total number of suppressor loci, superscript - indicates absence of the corresponding allele, and $\mathrm{Y}$ denotes the $\mathrm{Y}$ chromosome. Crosses between these individuals would give rise to $F_{1}$ males of genotype either $L^{-} / \mathrm{Y} ; n(S u) / n\left(S u^{-}\right)$or $L / Y ; n(S u) /$ $n\left(S u^{-}\right)$, where $L^{-}$males (lacking $\left.h m i-1\right)$ would be identified by their white phenotype.

Application of Markov chain models requires performing at least two consecutive backcrosses (BC1 and BC2) of $L^{-}$males (white males) with buzSL-Xd[hmi-1, $\left.w^{+}\right]$females (Fig. 2). For BC1, several individual crosses $(1 \times 1)$, hereafter referred to as "lines", involving white males taken from the $\mathrm{F}_{1}$ 's displaying the highest records of $w^{+}$male frequency were carried out. Again, $L^{-}$male offspring from these crosses can be genotypically described as $L^{-} / \mathrm{Y}$; $x(S u) / x\left(S u^{-}\right)$. The variable $x$ refers to the presence of an unknown number of suppressors in the male, $x \in(0, n)$. The same as before, several BC2 lines were obtained using white males selected from $\mathrm{BC} 1$ offsprings with the highest records of $w^{+}$male frequency. 
1 female buzSL-Xd[hmi-1, $\left.w^{+}\right] \quad x \quad 1$ male buzSL-5

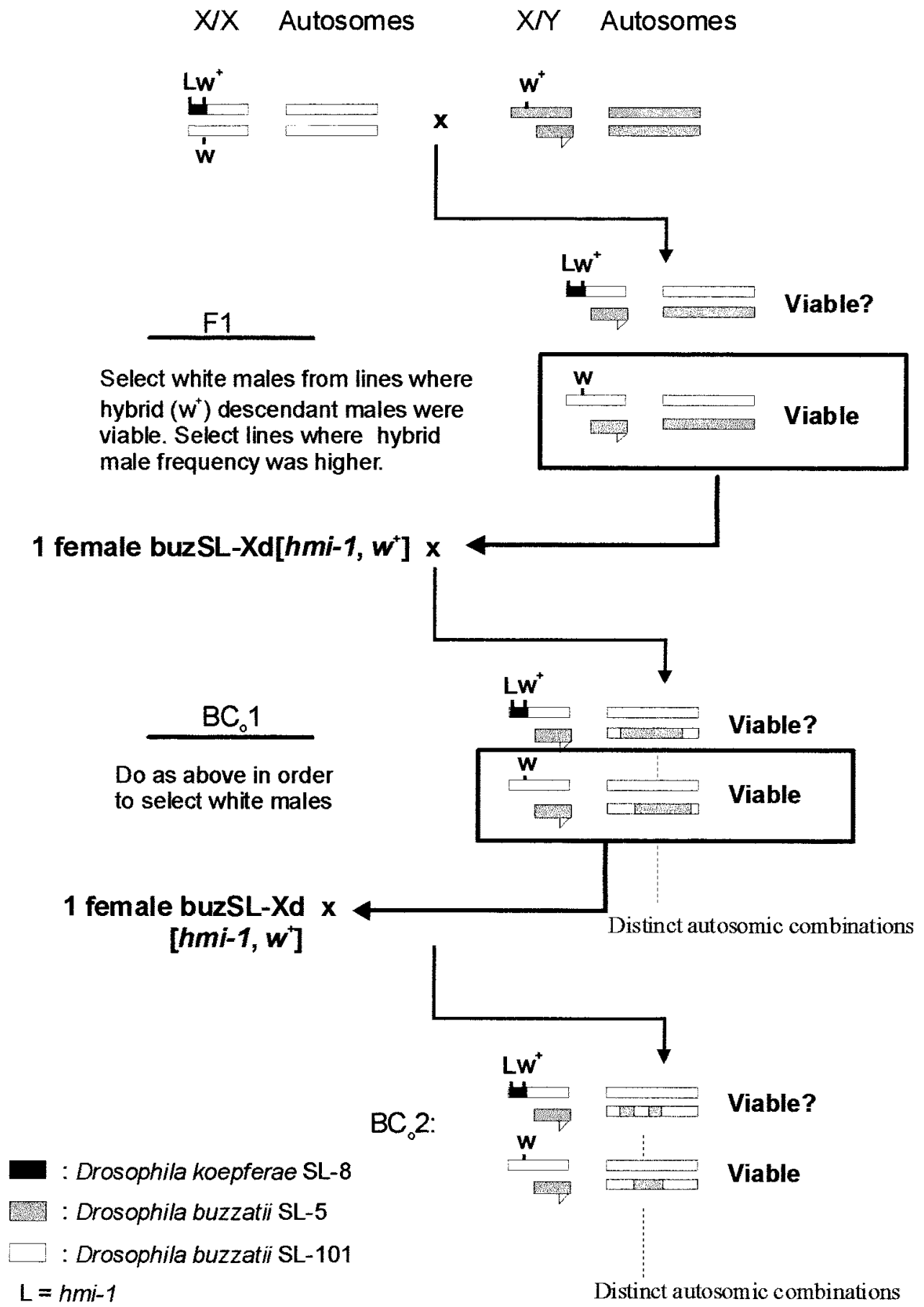

Fig. 1. Crossing protocol for application of Markov chain models to assess the number of buzSL-5 autosomes that are segregating to suppress the lethal effect of $h m i-1 . \quad \mathrm{BC}_{0}$ : offspring from backcrosses (1 and 2).

The model Consider Markov chain models $\left\{E_{0}, \ldots, E_{\mathrm{n}}\right\}$ where state $E_{\mathrm{i}}$ refers to $i$ suppressors in heterozygous condition $(0 \leq i \leq n)$ in the ( $\mathrm{BC} 1$ or $\mathrm{BC} 2)$ male progenitor. Note that the number of suppressors that are to be estimated actually corresponds to the number of autosomes carrying the suppressor effect, since the $\mathrm{X}$ chromosome is always from the $\mathrm{Xd}\left[h m i-1, w^{+}\right]$strain, and recombination between suppressors in the same chromosome is not possible with the crossing protocol described above since there is no crossing-over in male meiotic cells in these Drosophila species.

Transition probabilities between states $i$ and $j$ are given 
$\underline{\mathrm{BCl}}$

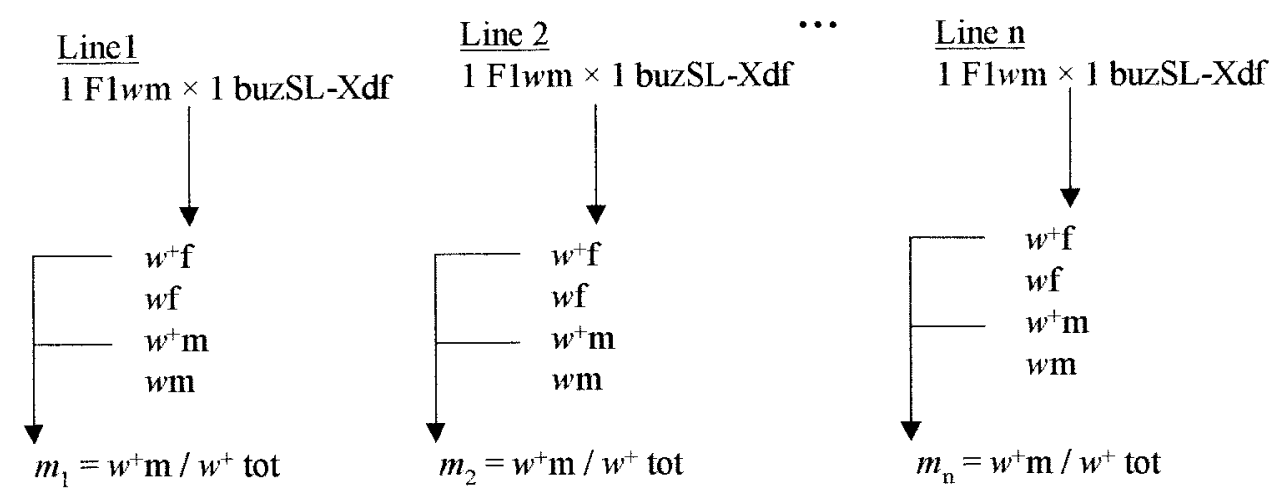

$p=$ number of lines with $m_{\mathrm{i}}$ corresponding to significative rescue / total $n$ lines

$\underline{\mathrm{BC} 2}$

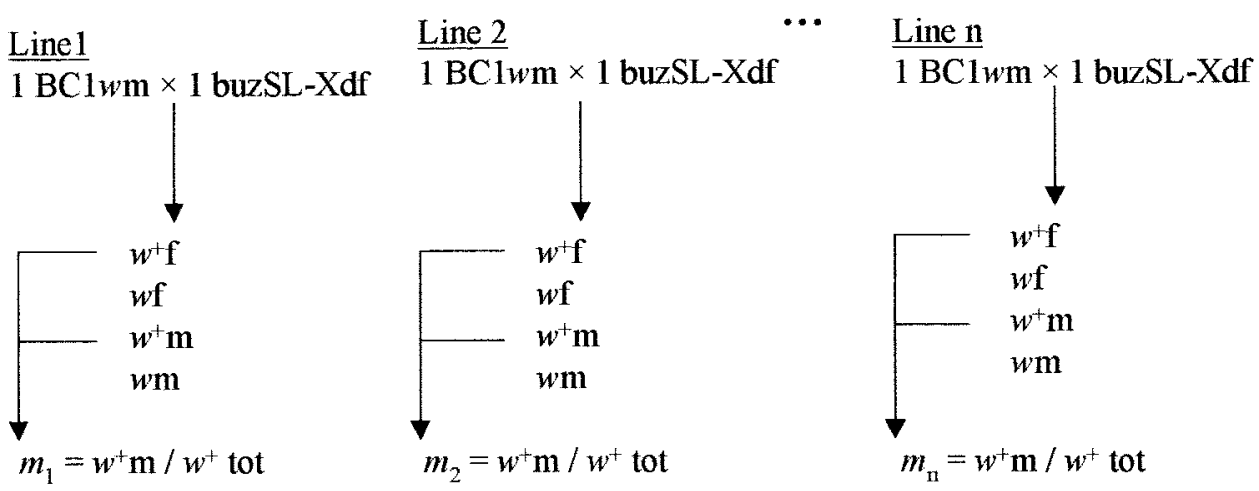

$p=$ number of lines with $m_{\mathrm{i}}$ corresponding to significative rescue / total $n$ lines

F1wm: F1 white male offspring; buzSL-Xdf: buzSL-Xd[hmi-l, $\left.w^{+}\right]$female; $w^{+}$: wild type female offspring from $\mathrm{BC} 1$ or $\mathrm{BC} 2$; wf: white female offspring from $\mathrm{BC} 1$ or $\mathrm{BC} 2 ; w^{+} \mathbf{m}$ : wild type male offspring from $\mathrm{BC} 1$ or $\mathrm{BC} 2$; wm: white male offspring from $\mathrm{BC} 1$ or $\mathrm{BC} 2$; $\mathrm{BC} 1$ wm: $\mathrm{BC} 1$ white male offspring; $\boldsymbol{w}^{+}$tot: wild type total per $\mathrm{BC} 1$ or $\mathrm{BC} 2$ line (males plus females).

Fig. 2. Crossing scheme showing how measures $m$ and $p$ are obtained both for $\mathrm{BC}_{0} 1$ and $\mathrm{BC}_{0} 2$.

by

$$
\gamma_{i j}=\left(\begin{array}{c}
i \\
j
\end{array}\right)(1 / 2)^{i}, \text { if } i \geq j \text {, and } \gamma_{i j}=0, \text { if } i<j ; i, j \in(0, n) \text {. }
$$

And the state distribution $\gamma^{(t)}=\left(\gamma_{0}{ }^{(t)}, \ldots, \gamma_{n}{ }^{(t)}\right)$ at any generation $t$ is

$$
\gamma^{(t)}=\gamma^{(0)} \wp \wp^{t}
$$

where $\gamma_{i}^{(t)}$ is the probability of state $i$ at generation $t, \gamma^{(0)}$ is the initial distribution of states, and $\wp=\left(\gamma_{i j}\right)$ is the matrix of transition probabilities.

We are interested in analysing two types of observed frequencies, which we will denote as $m$ and $p$ (Fig. 2). Let us define $m$ as the frequency of $w^{+}$(i.e., hybrid) males with respect to total $w^{+}$offspring (males + females). Let us define $p$ as the frequency of (BC1 or $\mathrm{BC} 2)$ lines where rescue of male viability is observed. Lines with male viability rescue are considered to be those where the relative frequency of $w^{+}$male offspring $(m)$ is significantly greater than 0.04. In order to obtain the corresponding expected values (parametric), $\mu$ and $\pi$, for any given number of suppressors, the possibility of epistasis must be considered. No epistasis means that any one suppressor, i.e. one chromosome, is enough to rescue $h m i-1$ males, so that the effect of the chromosome on the fitness of the male does not depend on the presence of other chromosomes; on the other hand, epistasis on fitness implies that one chromo- 
some requires the presence of some other chromosomes of the same strain in order to carry out the zygotic rescue of hmi-1 males.

A) Non-epistatic model

If we consider that no epistasis occurs, then the expected frequency $\pi$ of lines where rescue takes place are derived from:

$$
\pi=\sum_{i=1}^{n} \gamma_{i}^{(t)}=1-\gamma_{0}^{(t)}, 0 \leq \pi \leq 1
$$

For BC1 offspring, $t=0$; for BC2, $t=1$.

With respect to $\mu$, the probability that a male will inherit no suppressors is expressed by the transition probability $\gamma_{i 0}=(1 / 2)^{i}, i$ being the number of effective suppressors carried by the father. If $i=0$, then $96 \%$ of $w^{+}$males will be inviable. It follows that

$$
\mu=\left[1-0.96(1 / 2)^{i}\right] /\left[2-0.96(1 / 2)^{i}\right] .
$$

B) Epistatic model

If we assume that $r$ suppressors $(r>1)$ have to be present for rescue to occur, then:

$$
\pi=\sum_{i=r}^{n} \gamma_{i}^{(t)}
$$

where $n \geq r$ is the total number of suppressors.

The probability that a male will inherit an insufficient number of suppressors is:

$$
P_{\mathrm{inv}}=\sum_{j=0}^{r-1} \gamma_{i j}=\sum_{j=0}^{r-1}\left(\begin{array}{l}
i \\
j
\end{array}\right)(1 / 2)^{i}, \text { if } i \geq r ; P_{\mathrm{inv}}=1 \text {, if } i<r \text {. }
$$

Once again, $w^{+}$inviable males correspond to $0.96 \times P_{\text {inv }}$. Then,

$$
\mu=\left[1-0.96 \times P_{\text {inv }}\right] /\left[2-0.96 \times P_{\text {inv }}\right] .
$$

Data homogeneity was tested by means of the Pearson chi-squared goodness of fit statistic. The average value of $m$ was obtained as $\bar{m}=\sum_{1}^{k}=m_{i} / k$, where $k$ is the number of lines examined. The normal approximation to the binomial distribution for large sample sizes was applied for testing hypotheses concerning $\mu$ and $\pi$. Standard normal deviates $(z)$ were obtained without the correction for continuity (Therefore, e.g., $\left.z=(p-\pi) /(\pi(1-\pi) / k)^{1 / 2}\right)$. Tables of deviates were analysed by the sequential Bonferroni technique (Holm, 1979; Rice, 1989), under the composite null hypothesis $\left(\mathbf{H}_{0, \mathrm{c}}\right)$ that all the component deviates are taken from a standard normal distribution. Each deviate is replaced by its corresponding $P$ value, and ranked from smallest $\left(P_{1}\right)$ to largest $\left(P_{k}\right)$. If $P_{1} \leq \alpha / k$, where $\alpha$ is the selected significance level, the corresponding test is declared to indicate significance at the table wide $\alpha$ level. Successively higher $P$ values are similarly examined, until the inequality $P_{i} \leq \alpha /(1+k-i)$ is not met.

\section{RESULTS AND ANALYSIS}

$\mathrm{F}_{1}$ offsprings from individual crosses between buzSL$\mathrm{Xd}\left[\mathrm{hmi}-1, \mathrm{w}^{+}\right]$females and males from strains buzSL-5(1) and (2), buzSL-In(4)s, buzDF, and buzM rendered $\bar{m}$ values significantly higher than the expected 0.04 (table 1), Therefore evidencing the presence of suppressors of the hmi-1 effect.

Different lines from buzSL-5 (1) $\mathrm{F}_{1}$ crosses were chosen to carry out the analysis by Markov chains, both because the frequency of $w^{+}$males was significantly higher than 0.04 in all the lines examined (data not shown although in table 1 it is shown that such data was homogeneous), and their average was the highest ( $\bar{m}=0.41$ in table 1 ).

From the $\mathrm{F}_{1}$ of crosses with buzSL-5 (1), two backcross generations (BC1 and $\mathrm{BC} 2$ ) were obtained (see crossing protocol). The results of the two backcrosses are shown in tables 2 and 4 (BC1 and BC2 respectively) in terms of the frequency $m$ of $w^{+}$males. To build the second backcross, male parents were chosen from the BC1 line dis-

Table 2. Observed frequencies of $w^{+}$males $(m)$ in BC1 offsprings. The male parents coming from the $\mathrm{F}_{1}$ cross produced by buzSL-5(1) in Table 1. Sample size is the total number of $w^{+}$(i.e., hybrid) offspring recorded. Standard deviates of the normal approximation to the binomial distribution, under different null hypotheses $\left(\mathbf{H}_{\mu=\mu 0}\right)$, are indicated for each line. $*$ significant departures from the null hypothesis at the tablewide 0.05 level, after sequential Bonferroni tests.

\begin{tabular}{ccccccc}
\hline \hline Line & $m$ (sample size) & $Z_{\mu=0.04}$ & $Z_{\mu=0.22}$ & $Z_{\mu=0.14}$ & $Z_{\mu=0.25}$ & $Z_{\mu=0.34}$ \\
\hline 1 & $0.14(66)$ & $4.15^{*}$ & -1.57 & 0.00 & -2.06 & $-3.43^{*}$ \\
2 & $0.12(93)$ & $3.94^{*}$ & -2.33 & -0.56 & $-2.89^{*}$ & $-4.48^{*}$ \\
3 & $0.08(60)$ & 1.58 & $-2.62^{*}$ & -1.34 & $-3.04^{*}$ & $-4.25^{*}$ \\
4 & $0.23(40)$ & $6.13^{*}$ & 0.15 & 1.64 & -0.29 & -1.47 \\
5 & $0.26(39)$ & $7.01^{*}$ & 0.60 & 2.16 & 0.14 & -1.05 \\
6 & $0.20(66)$ & $6.63^{*}$ & -0.39 & 1.40 & -0.94 & $-2.40^{*}$ \\
7 & $0.18(99)$ & $7.11^{*}$ & -0.96 & 1.15 & -1.61 & $-3.36^{*}$ \\
8 & $0.38(16)$ & $6.94^{*}$ & 1.54 & $2.77^{*}$ & 1.20 & 0.34 \\
9 & $0.09(65)$ & $2.06^{*}$ & $-2.53^{*}$ & -1.10 & $-2.98^{*}$ & $-4.25^{*}$ \\
\hline
\end{tabular}


playing the highest rescue value (line 8 , i.e. $\mathrm{BC} 1-8$, in Table 2). Standard normal deviates $(z)$ of the approximation to the binomial distribution, under different null hypotheses, and the results of testing those hypothesis against observed values, are also shown (see later).

Almost all BC1 lines displayed a significant rescue of adult hybrid males at the table-wide 0.05 level, since all $P$ values (except $P_{9}$ corresponding to line 3 ) of $z$ deviates met the inequality $P_{\mathrm{i}} \leq 0.05 /(1+k-i)$ of the sequential Bonferroni test (Table $2,3^{\text {rd }}$ column; $k=9$ ). With respect to line $3, P_{9}=0.057$ which is marginally significant. Therefore, we consider that observed frequency of viability lines $p$ belongs to [0.88-1] interval . Observed values for $m$ ranged from 0.08 to 0.38 ( $\bar{m}=0.16)$, the heterogeneity among lines being significant $\left(\chi_{\mathrm{H}}^{2}=16.77^{*}, 8 \mathrm{df}, 0.05>\right.$

Table 3. Expected frequencies of hybrid males $(\mu)$ in the offspring of test crosses (BC1 or $\mathrm{BC} 2$, see Material and Methods), for different epistatic models. A maximum of 5 autosomal suppressors (one per autosome) of the $h m i-1$ lethal factor is assumed to be in heterozygous condition in the tested genotype (always the male sex, according to our protocol); the transimission of any combination of at least $r$ of these factors brings about full rescue of adult $h m i-1$ hybrid males in the offspring. $E_{i}$ states refer to $i$ suppressors in heterozygous condition in the male progenitor.

\begin{tabular}{cll||ccc}
\hline \hline \multicolumn{3}{c||}{ Epistatic model } & \multicolumn{1}{c||}{$\mu$} & \multicolumn{3}{c}{ Epistatic model } & \multirow{2}{*}{$\mu$} \\
$E_{i}$ & $r$ & & $E_{i}$ & $r$ & \\
\hline 5 & 5 & 0.065 & 4 & 3 & 0.25 \\
5 & 4 & 0.18 & 4 & 2 & 0.41 \\
5 & 3 & 0.34 & 3 & 3 & 0.14 \\
5 & 2 & 0.45 & 3 & 2 & 0.34 \\
4 & 4 & 0.09 & 2 & 2 & 0.22 \\
\hline
\end{tabular}

$P>0.01)$.

Both under non-epistatic and epistatic models, the observed value for $p$ at BC1 means that the frequency of effective suppressor in strain buzSL-5 is high. The expected value $\pi$ at BC1 depends on distribution of suppressors at $\mathrm{F} 1$ and hence at parental strains. If supressors are fixed or almost fixed in parental strains then we expect roughly a value near 1 independently of the model we consider. The observed $p$ value in $\mathrm{BC} 1$ seems to sustain this. However, the best fit to observed rescue frequencies within lines $(m)$ with the non-epistatic model is obtained with just 1 suppressor $(\mu=0.34)$, and then still only three out of nine introgression lines (numbers 4, 5, and 8 in Table 2, last column) do not significantly differ from the expected rescue frequency $(\mu=[1-0.96(1 /$ $2)] /[2-0.96(1 / 2)]=0.34$, according to $(4))$, since there are simply too few hybrid males in most lines. Therefore, lines $1,2,6,7,9$ in table 2 allow the rejection of all non epistatic models $(\mu \geq 0.34)$. Non-redundant epistatic models $(n=r)$ provide a much better fit to observed rescue frequencies, both for two $(\mu \cong 0.22)$ and three $(\mu \cong 0.14)$ interacting supressors (see Table $2,4^{\text {th }}$ and $5^{\text {th }}$ columns, respectively; see Table 3 for expected frequencies under different epistatic models), although a few lines depart significantly from expectations, reflecting the significant heterogeneity observed in $m$ values among BC1 lines. Therefore, line 9 of table 2 allows the rejection of $\mu=0.22$ ( $n=r=2$ model) and line 8 of the same table allows the rejection of $\mu=0.14$ ( $n=r=3$ model). This heterogeneity is easily explained if redundant epistatic models $(n>r)$ apply, simply by the expected segregation of supressors among F1 offspring males (i.e. male parents in BC1 cross). Therefore, if five suppressors (the maximum number we can detect with our experimental approach) inter-

Table 4. Observed frequencies of $w^{+}$males $(m)$ in BC2 offsprings from the $\mathrm{BC} 1$ cross produced by line 8 in Table 2 . Sample size is the total number of $w^{+}$(i.e., hybrid) offspring recorded. Standard deviates of the normal approximation to the binomial distribution, under different null hypotheses $\left(\mathbf{H}_{\mu=\mu_{0}}\right)$, are indicated for each line. * $=$ significant departures from the null hypothesis at the table-wide 0.05 level, after sequential Bonferroni tests.

\begin{tabular}{ccccccc}
\hline \hline Line & $\mathrm{m}$ (sample size) & $\mathrm{Z}_{\mu=0.04}$ & $\mathrm{Z}_{\mu=0.22}$ & $\mathrm{Z}_{\mu=0.14}$ & $\mathrm{Z}_{\mu=0.25}$ & $\mathrm{Z}_{\mu=0.34}$ \\
\hline 1 & $0.07(41)$ & 0.98 & -2.32 & -1.29 & $-2.66^{*}$ & $-3.65^{*}$ \\
2 & $0.07(57)$ & 1.16 & $-2.73^{*}$ & -1.52 & $-3.14^{*}$ & $-4.30^{*}$ \\
3 & $0.00(43)$ & -1.34 & $-3.48^{*}$ & $-2.65^{*}$ & $-3.79^{*}$ & $-4.71^{*}$ \\
4 & $0.07(82)$ & 1.39 & $-3.28^{*}$ & -1.83 & $-3.76^{*}$ & $-5.16^{*}$ \\
5 & $0.21(146)$ & $9.87^{*}$ & -0.29 & 2.44 & -1.12 & $-3.32^{*}$ \\
6 & $0.14(37)$ & $3.10^{*}$ & -1.17 & 0.00 & -1.54 & $-2.57^{*}$ \\
7 & $0.00(24)$ & -1.00 & $-2.60^{*}$ & -1.98 & $-2.83^{*}$ & $-3.52^{*}$ \\
8 & $0.08(13)$ & 0.73 & -1.22 & -0.62 & -1.41 & -1.98 \\
9 & $0.19(27)$ & $3.98^{*}$ & -0.63 & 0.75 & -0.72 & -1.64 \\
10 & $0.17(29)$ & $3.57^{*}$ & -0.65 & 0.47 & -0.99 & -1.93 \\
11 & $0.10(39)$ & 1.91 & -1.81 & -0.72 & -2.16 & $-3.16^{*}$ \\
12 & $0.30(45)$ & $8.90^{*}$ & 1.29 & $3.09^{*}$ & 0.77 & -0.57 \\
13 & $0.27(56)$ & $8.78^{*}$ & 0.90 & $2.80^{*}$ & 0.35 & -1.33 \\
\hline
\end{tabular}


acting in groups of any three are segregating among F1 offspring males, three different $m$ values (for 5,4 and 3 suppressors inherited) are expected in BC1 lines (see Table $3, \mu=0.34$ for state $E_{5}, \mu=0.25$ for $E_{4}$, and $\mu=0.14$ for $E_{3}$ ). Neither of these frequencies alone can explain BC1 results (see Table 2, three last columns, for tests of the corresponding hypotheses), but their combination easily can.

In contrast to $\mathrm{BC} 1$ lines, a significant rescue of adult hybrid males was observed only in 6 out of 13 BC2 offsprings $\left(p=0.46\right.$, Table $4,3^{\text {rd }}$ column). Hybrid male frequencies $(m)$ in these six lines ranged from 0.14 to 0.30 $(\bar{m}=0.21) . \quad \chi_{\mathrm{H}}^{2}=5.09,5 \mathrm{df}, P=0.4$.

Since all male parents (white phenotype) for BC2 crosses came from the same BC1 offspring (BC1-8), there should be no heterogeneity in the initial distribution of states, so that (2):

$$
\gamma^{(1)}=\gamma^{(0)} \wp^{1}=(0, \ldots, 0,1) \wp=\left(\gamma_{n 0}, \ldots \gamma_{n r}, \ldots \gamma_{n n}\right),
$$

where $n$ corresponds to the total number of suppressors borne in heterozygosis by the grandfather (the white male that fathered $\mathrm{BC} 1-8$ ), and $\gamma_{n j}$ refers to the transition probabilities from state $n$ to $j$. If, as before, $r$ designates the minimum number of suppressors that should be present in the genome of a hybrid male for it to be viable as adult, then at BC2 the expected frequency of lines displaying significant rescue activity (5) would be given simply by:

$$
\pi=\sum_{j=0}^{n} \gamma_{n j}-\sum_{j=0}^{r-1} \gamma_{n j}=1-\sum_{j=0}^{r-1} \gamma_{n j}
$$

Table 5 shows the results of testing different epistatic models against the observed value of $p(0.46)$. First, for

Table 5. Expected frequencies of viability rescue lines $(\pi)$ obtained after a test cross (see Material and Methods), for different kinds of redundant epistatic models $(n>r)$ models. $E_{n}$ states refer to $n$ suppressors in heterozygous condition in the tested genotype (the grandfather of all the offsprings examined in the lines); any combination of at least $r$ of these factors brings about full rescue of $h m i-1$ hybrid males. The goodness of fit between these expected frequencies and the experimental value obtained from $\mathrm{BC} 2$ lines in Table $4(p=0.46)$ is also shown.

\begin{tabular}{cccc}
\hline \hline \multicolumn{2}{c}{ Epistatic model } & $\pi_{0}$ & $\chi^{2}{ }_{\pi=\pi}, 1 \mathrm{df}$ \\
$E_{n}$ & $r$ & & \\
\hline 5 & 4 & 0.19 & $4.59^{*}$ \\
5 & 3 & 0.50 & $\approx 0 \mathrm{~ns}$ \\
5 & 2 & 0.81 & $8.12^{* *}$ \\
4 & 3 & 0.31 & $0.78 \mathrm{~ns}$ \\
4 & 2 & 0.69 & $2.19 \mathrm{~ns}$ \\
3 & 2 & 0.50 & $\approx 0 \mathrm{~ns}$ \\
\hline
\end{tabular}

$\mathrm{ns}: P>0.05 ; *: 0.05>P>0.01 ; * *: 0.01>P>0.001$ non-redundant models, the highest expected frequency of rescue lines is obtained for two interacting chromosomes ( $n=r=2$ ), namely $\pi=0.25$, which is slightly significant with respect to the observed value ( $z=1.75, P=0.04$ ). By other side the expected $m$ value under this model is, from (6) and (7) with $i=2, m=0.22$ which fits well with all rescue lines in table 4 (lines $5,6,9,10,12,13$ ) but as we saw falls in line 9 (and line 3) of BC1 lines (table 2). Also, lines 12,13 in table 4 allow the rejection of $\mu=0.14(n=r$ $=3$ model) and lines 5,6 in the same table allow the rejection of all non epistatic models $(\mu \geq 0.34)$. Thus, although we can not discard a sample size effect with respect the hypothesis $n=r=2$, a better explanation of our data comes with $n>r$ redundant epistatic systems. Four different cases of combinations of $n$ factors taken $\geq r$ at a time $\left(\mathbf{C}_{(n}\right.$, $\geq r)$ ) were not significant at a table-wide 0.05 level, namely $(5, \geq 3),(4, \geq 3),(4, \geq 2)$, and $(3, \geq 2)$ (see Table 5$)$. As to rescue frequencies within BC2 lines $(m)$, all observations can be explained by any of these combinations (Table 4). However, if lines from $\mathrm{BC} 1$ and $\mathrm{BC} 2$ are taken altogether, only the expectations from $\mathbf{C}_{(5, \geq 3)}$ and $\mathbf{C}_{(4, \geq 3)}$ are always met, whereas $\mathbf{C}_{(4, \geq 2)}$ and $\mathbf{C}_{(3, \geq 2)}$ fail to explain the observations from lines 3 and 9 in $\mathrm{BC} 1$.

\section{DISCUSSION}

In this work the polymorphism for hybrid male rescue factors has been investigated in a series of $D$. buzzatii stocks, both from Argentina and Spain, after experimental crosses with an introgression line bearing a hybrid-lethal factor from its sibling D. koepferae. Among spanish stocks only a single one (buz M, table 1) showed a significant, although slight, viability rescue effect. Stocks from Argentina displayed strong differences in their hybrid male rescue activity. Therefore, buzSL5(1) had the highest effect (all replicates produced hybrid male rescue), buzSL-In(4)s had a partial effect (significant increase of hybrid male viability was detected only in a few replicates), whereas buzSL-In(5)1 and buzSL6 had no effect at all. These differences indicate the probable existence of polymorphism for hybrid male rescue factors in the natural population of San Luis, where the founding flies of these stocks were sampled. This polymorphism may have been lost during colonisation of the Old World by $D$. buzzatii (this species originated in South America, and then was spread to the Old World and Australia; Ruiz et al, 1982).

The existence of a polymorphism for rescue factors in $D$. buzzatii means that the effect of the introgression of the hybrid lethal factor $h m i$-1from $D$. koepferae will depend on the genetic background. Examples of conditional viability have long been known in Drosophila, such as can be found in the works by Sturtevant (1929), Watanabe et al. (1977) and Lee (1978), where the viability of hybrid females from the cross between $D$. melanogaster females 
and $D$. simulans males is shown to vary according to temperature and genetic background. Recently, it has been reported that a survey of approximately $50 \%$ of $D$. simulans genome rendered no regions that, in hemizygosis, bring about unconditional inviability of hybrids with $D$. melanogaster (Coyne et al., 1998); several regions were shown to have a deleterious effect on hybrid viability, but in every case this effect was found to disappear after changing the culture temperature or the $D$. simulans genetic background. In contrast to these findings in $D$. simulans $\mathrm{x} D$. melanogaster hybrids, no effect of temperature on hybrid viability was observed in our case (data not shown).

It can be argued that our results are biased since we select for $\mathrm{BC} 1$ and $\mathrm{BC} 2$ the lines with highest rescue. However the aim of the work is precisely to show the dynamical and highly polymorphic condition of buzzatiikoepferae viability hybrid systems. We do not say that all buzzatii SL-5 individuals have $n \geq r$ viability rescue factors, we only say, and show, that these kind of systems probably exist in this buzzatii strain. Our results for the hmi-1 system discard the existence of what we call a nonepistatic model of $h m i$ - 1 suppressor effect i.e. we show that more than one chromosome with suppressor effect is needed for rescue to occur. Our data are best interpreted by assuming that factors from at least three $D$. buzzatii autosomes, and even perhaps from all four of them, make a contribution to hybrid male rescue, but with a considerable degree of functional redundancy, that is, "more or less strongly overlapping gene functions" (Tautz, 1992). This functional redundancy could easily explain the observed discrepancy between the $\%$ of lines displaying viability rescue and the $\%$ of viable hybrids within each line, on the one hand, and the heterogeneity in rescue effect of the examined stocks, on the other. Here it is worth mentioning that in a previous work (Carvajal et al., 1996) it was shown that apparently two interacting factors from $D$. koepferae had to be cointrogressed with $h m i-1$ for hybrid males to be viable. Although later unpublished evidence led us to the conclusion that actually just one of these factors (mapped to 4D1-D5) is enough to bring about full rescue of $h m i-1$ males. How can this information from $D$. koepferae (1 complementary factor is enough for rescue) be reconciled with that from $D$. buzzatii (different interacting sets, combining factors from different autosomes)? First of all, it must be reported that preliminary data indicate the existence of other complementary factors of $\mathrm{hmi}$ 1 in $D$. koepferae, independent from the factor located in 4D1-D5. Then, it may be that the evolutionary divergence of these factors between $D$. koepferae and $D$. buzzatii has come to a different state in the different populations, corresponding to somewhat different efficiencies in their ability to interact with $h m i$-1, therefore leading to a "dosage" effect on the rescue of hybrid male viability.

It is also worth mentioning that fluctuating asymmetry, typically associated to developmental instabilities (Mфller \& Swaddle, 1997), has also been observed in interespecific hybrids (Carvajal, 1996; Wade et al., 1997) and consistently actually appear in several buzzatii-koepferae introgression lines (H. Naveira personal communication). It could well be the case that the systems of complementary genes whose disruption brings about hybrid inviability consist of regulators occupying relatively lower levels in the developmental hierarchy, which usually manifest functional redundancy (Krumlauf, 1992).

Finally, it must be stressed that the estimate of the number of lethal-suppressors obtained in this paper should be confirmed by appropriate crosses using chromosome markers. Those results constitute simply an estimate of the minimum number of linkage-groups affecting the character (hybrid male rescue), and do not give information on the nature of the genetic factors involved (either polygenes or major genes). Anyway, it must be remembered that hybrid inviability factors reported so far generally correspond to major genes, whereas most sterility factors behave as polygenes (Maside et al., 1998).

I thank Horacio Naveira for many comments on the original manuscript. My research was supported by a scholarship from Universidade da Coruña, and grants 10305B95, 10304B97 and PGIDT 99BIO10302 from the "Xunta de Galicia" to H. N.

\section{REFERENCES}

Carson, H. L., and Templeton, A. R. (1984) Genetic revolutions in relation to speciation phenomena: The founding of new populations. Ann. Rev. Ecol. Syst. 15, 97-131.

Carvajal, A. R. (1996) Developmental disruptions in hybrids between $D$. buzzatii and D. koepferae. Int. J. Dev. Biol. Supplement 1, 93-94.

Carvajal, A. R., Gandarela, M. R., and Naveira, H. F. (1996) A three locus system of interspecific incompatibility underlies male inviability in hybrids between $D$. buzzatii and $D$. koepferae. Genetica 98, 1-19.

Coyne, J. A., Simeonidis, S., and Rooney, P. (1998) Relative paucity of genes causing inviability in hybrids between Drosophila melanogaster and D. simulans. Genetics 150, 10911103.

Fontdevila, A., Pla, A., Hasson, E., Wasserman, M., Sánchez, A. Naveira, H., and Ruiz, A. (1988) Drosophila koepferae: A new member of the Drosophila serido (Diptera: Drosophilidea) superspecies taxon. Ann. Entomol. Soc. Am. 81, 380-385.

Holm, S. (1979) A simple sequentially rejective multiple test procedure. Scand. J. Stat. 6, 65-70.

Hutter, P., Roote, J., and Ashburner, M. (1990) A genetic basis for the inviability of hybrids between sibling species of Drosophila. Genetics 124, 909-920.

Krumlauf, R. (1992) Evolution of the vertebrate Hox Homeobox genes. ByoEssays 4, 245-252.

Lee, W. H. (1978). Temperature sensitive viability of hybrid between Drosophila melanogaster and D. simulans. Jpn. J. Genet. 53, 339-344.

Maside, X. R., Barral, J. P., and Naveira H. F. (1998) Hidden effects of $\mathrm{X}$ chromosome introgressions on spermatogenesis in Drosophila simulans $\times$ D. mauritiana hybrids unveiled by interactions among minor genetic factors. Genetics $\mathbf{1 5 0}$, 
$745-754$.

Møller, A. P., and Swaddle J. P. (1997) Asymmetry, developmental stability, and evolution. Oxford Univ. Press, Oxford, U.K.

Naveira, H. F., and Fontdevila, A. (1985) The evolutionary history of $D$. buzzatii IX. High frequencies of new chromosome rearrangements induced by introgressive hybridization. Chromosoma 91, 87-94.

Naveira, H. F., and Fontdevila, A. (1991) The evolutionary history of D. buzzatii XXII. Chromosomal and genic sterility in male hybrids of $D$. buzzatii and $D$. koepferae. Heredity 66 , 233-239.

Naveira, H. F., Pla, C., and Fontdevila, A. (1986) The evolutionary history of Drosophila buzzatii XI. A new method for citogenetic localisation based on asynapsis of polytene chromosomes in interspecific hybrids of Drosophila. Genética 71, 199-212.

Nei, M., and Zhang, J. (1998) Molecular origin of species. Science 282, 1428-1429.

Rice, W. R. (1989) Analyzing tables of statisticals tests. Evolution 43, 223-225.
Ruiz, A., and Fontdevila, A. (1981) Two new chromosome arrangements in Drosophila buzzatii. DIS 56, 111-114.

Ruiz, A., Fontdevila, A., and Wasserman, M. (1982) The evolutionary history of D. buzzatii. III. Cytogenetic relationships between two sibling species of the buzzatii cluster. Genetics 101, 503-518.

Sturtevant, A. H. (1929) The genetics of Drosophila simulans. Carnegie Inst. Washington Publ., 399, 1-62.

Tautz, D. (1992) Redundancies, development and the flow of information. ByoEssays 4, 263-265.

Wade, M. J. Johnson, N. A. Jones, R. Siguel, V., and Mcnaughton, M. (1997) Genetic variation segregating in natural populations of Tribolium castaneum affecting traits observed in hybrids with $T$. freemani. Genetics 147, 1235-1247.

Watanabe, T. K., Lee, W. H., Inoue, I., and Kawanishi, M. 1977. Genetic variation of the hybrid crossability between Drosophila melanogaster and Drosophila simulans. Jpn. J. Genet. 52, 1-8.

Wu, C-I., and Palopoli, M. F. (1994) Genetics of postmating reproductive isolation in animals. Annu. Rev. Genet. 28, 283-308. 